\title{
Prevalence and treatment follow-up of drug-resistant extra-pulmonary tuberculosis in rural communities in Narshingdi, Bangladesh
}

\author{
Hafsa Afroz ${ }^{1}$, Md. Azhar Ali ${ }^{1,2}$, Md. Fakruddin ${ }^{3}$, Kamrunnahar $^{1}$, Suvamoy Datta $^{1}$
}

${ }^{1}$ Department of Microbiology, Primeasia University, Dhaka, Bangladesh
${ }^{2}$ Department of Microbiology, Upazilla Health Complex, Palash, Narsingdi, Bangladesh
${ }^{3}$ Industrial Microbiology Laboratory, IFST, BCSIR, Dhaka, Bangladesh

Received: 19 June 2014

Accepted: 5 July 2014

\section{*Correspondence:}

Hafsa Afroz,

E-mail: hafsa.afroz@gmail.com

Copyright: (C) the author(s), publisher and licensee Medip Academy. This is an open-access article distributed under the terms of the Creative Commons Attribution Non-Commercial License, which permits unrestricted non-commercial use, distribution, and reproduction in any medium, provided the original work is properly cited.

\begin{abstract}
Tuberculosis is a chronic communicable disease. Infections are initially asymptomatic and latent but eventually progresses to active disease, which, if left untreated, may have $\geq 50 \%$ mortality. In 2011 , an anti-tuberculosis drug resistance survey in Narsingdi, Bangladesh enrolled both new and retreatment patients. In the study, a number of villages were randomly selected and 152 extra pulmonary tuberculosis (EPTB) patients were identified. All the patients received treatment with standardized first-line drug (FLD) regimens and were investigated to document treatment efficiency. Among the patients, 34 were reported in lower income, 112 in medium income and only 06 in higher income group. Farmers (16.4\%) in low income group, and female housewives (41.4\%) in medium income group were mostly infected. Silicosis and malnutrition were assumed responsible for high EPTB cases in farmers and housewives respectively. The working youth groups (25-34) were found most vulnerable. Extra pulmonary TB sites of infection was found pleural TB $29.6 \%$ patients, glands TB $24.3 \%$ patients, abdominal TB $21.0 \%$ patients, spinal TV $19.7 \%$ patients, tubercular meningitis $436 \%$ patients \& tubercular pericarditis $0.65 \%$ patient. Multi-drug resistant (MDR) EPTB was detected in both new and retreatment patients. Patients with MDR-EPTB who had been declared cured with first-line anti-TB treatment had a high rate of TB recurrence and death. The rate of TB recurrence and death was high four years after MDR-TB patients were judged to have been cured. PTB patients in Bangladesh had high recurrence and death rates even after treatment with standardized FLD regimens, reinforcing the need for early survey, diagnosis and treatment, including assessment of treatment outcomes.
\end{abstract}

Keywords: Age, Bangladesh, Pulmonary tuberculosis, Socio-economic condition, Treatment efficiency

\section{INTRODUCTION}

Tuberculosis is a common and in many cases lethal infectious disease caused by various strains of Mycobacterium spp., usually Mycobacterium tuberculosis. ${ }^{1}$

Most infections in humans result in an asymptomatic, latent infection and about one in ten latent infections eventually progresses to active disease. The mortality rate for untreated tuberculosis is more than $50 \%$. One third of the world's population is thought to have infected and new infections occur at a rate of about one of two. ${ }^{2}$

Extra-pulmonary tuberculosis (EPTB) refers to tuberculosis (TB) outside the lungs. Among TB infections, EPTB involvement accounts for up to onethird of all cases. ${ }^{3}$ The most common sites of extrapulmonary $\mathrm{TB}$ are lymph nodes, genitourinary tract, pleura, bones and joints, meninges and the central nervous system, peritoneum and other abdominal organs. ${ }^{4}$ The diagnosis of extra-pulmonary tuberculosis poses a 
special challenge as it is often missed or misdiagnosed due to its atypical presentations. The first step in its diagnosis is its awareness and a high index of suspicion by the physicians. Bangladesh is a high TB burden area with 300000 new cases and 45000 deaths per year. However, to best of our knowledge, no bacteriological data regarding EPTB has been published in Bangladesh. ${ }^{5}$ The propagation of tuberculosis is strongly related to socioeconomic status and hygiene condition of the patient. ${ }^{6}$ Patients with extra-pulmonary TB do not receive specific attention in international TB control strategies. However, extra-pulmonary TB contributes significantly to TB-related morbidity and can cause complications, lifelong sequelae and disabilities. From a public health perspective, there is therefore a need to address this group of patients, as they do contribute to the total burden of disease and they do have a significant impact on available resources of national health systems. ${ }^{7}$

One of the important challenges for TB control is drug resistance, particularly multidrug resistant TB. Treatment is difficult and requires long courses of multiple antibiotics. Multi-drug-resistant tuberculosis threatens the treatment regimen further. ${ }^{8}$ Treatment for TB consist multiple antibiotic regimen. Effective TB treatment is difficult, due to the unusual structure and chemical composition of the mycobacterial cell wall, which makes many antibiotics ineffective and hinders the entry of drugs. ${ }^{9}$ TB requires much longer periods of treatment (around 6 to 24 months) to entirely eliminate mycobacteria from the body ${ }^{10}$ instead of the short course of antibiotics typically used. Latent TB treatment usually uses a single antibiotic, while active TB disease is best treated with combinations of several antibiotics, to reduce the risk of the bacteria developing antibiotic resistance. ${ }^{11}$ Drug-resistant TB is a public health issue in many developing countries, as treatment is longer and requires more expensive drugs. Multi-drug-resistant tuberculosis (MDR-TB) is defined as resistance to the two most effective first-line TB drugs: rifampicin and isoniazid. ${ }^{12}$

Considering the above facts, the aim of this study was to assess the prevalence of pulmonary tuberculosis in Palash, Narsingdi, Bangladesh. The study also aims at evaluation of drug resistant pattern and treatment outcome with standard drugs.

\section{METHODS}

\section{Ethics statement}

The study was approved by the Upazilla health complex, Palash, Narsingdi ethical committee for TB operational research. Written informed consent was obtained from individuals before an interview was conducted and sputum samples were collected. If the individual had died, written informed consent was obtained from the family member before they were interviewed. Patients and TB hospitals were informed of the sputum smear, culture, and DST test result. MDR-TB patients identified during follow-up were requested to go to the TB hospital for treatment, including with second-line drugs.

\section{Drug resistance survey}

For the drug resistance survey (DRS), the "Guidelines for surveillance of drug resistance in tuberculosis" developed by WHO/IUATLD were followed. ${ }^{13}$ In the district Narsingdi, a number of villages were randomly selected under Palash Union council for the survey. All smearpositive cases in the survey area during the study period (From February to November, 2011) were diagnosed and positive samples were eligible for inclusion. ${ }^{14}$ Drug resistance was assessed against four anti-tuberculosis drugs such as isoniazid, rifampicin, streptomycin and ethambutol.

\section{Treatment regimen and outcome assessment}

The district Narsingdi followed the National Tuberculosis Programme (NTP) treatment guidelines for all TB patients. Treatment of new smear-positive patients consisted of 2 months of isoniazid, rifampicin, pyrazinamide, and ethambutol followed by 4 months of isoniazid and rifampicin three times weekly $\left(2 \mathrm{H}_{3} \mathrm{R}_{3} \mathrm{Z}_{3} \mathrm{E}_{3} / 4 \mathrm{H}_{3} \mathrm{R}_{3}\right)$. Patients who had previously received at least one month of TB treatment (i.e. retreatment patients) received 2 months of isoniazid, rifampicin, pyrazinamide, streptomycin and ethambutol, followed by 6 months of isoniazid, rifampicin, and ethambutol three times weekly $\left(2 \mathrm{H}_{3} \mathrm{R}_{3} \mathrm{Z}_{3} \mathrm{E}_{3} \mathrm{~S}_{3} / 6 \mathrm{H}_{3} \mathrm{R}_{3} \mathrm{E}_{3}\right.$. $)$. Drug intake was observed at the health facility throughout the treatment.

Treatment outcomes were assessed using international definitions and were recorded in routine treatment registers. ${ }^{15}$ Treatment success was defined as either cure or treatment completed. Cure was defined as a patient who was sputum smear-negative in the last month of treatment and on at least one previous occasion. Treatment completed was defined as a patient who had completed treatment but who did not meet the criteria to be classified as a cure or a failure. Treatment failure was defined as a patient who was sputum smear-positive at 5 months or later during treatment. Default was defined as a patient whose treatment had been interrupted for two consecutive months or more. Transfer out was defined as a patient who had been transferred to another recording and reporting unit and hence treatment outcome was unknown. Death was defined as a patient who died for any reason during the course of treatment. The cause of death during TB treatment was not recorded. ${ }^{16}$

\section{Follow up of EPTB patients}

Staffs from regional TB centers included in the DRS were instructed on how to follow-up the 264 patients included in the DRS. Regional staffs and one provincial staff member interviewed patients in their home in the period. During the interview, participants were asked 
about presence of common EPTB symptoms (i.e., cough, back pain, chest pain, fever, fatigue, anorexia and night sweat, weight loss). Blood samples were collected from all participants for microscopy, culture and DST using the same methods. Staff rechecked the sample examination results, diagnosis and dates with the medical files in the facility. If the patient had died, family members were interviewed about the time of death and its probable cause. Staff would recheck the cause with the death certificate provided by the hospital. Patients who had TB registered as a cause of death were assumed to have had recurrent $\mathrm{TB} .^{17}$

\section{Data management and analysis}

Analysis was performed using SPSS 13.0 (SPSS Inc. Chicago, IL, USA). For the analysis of treatment outcome from initial TB treatment, we compared patients who were cured to those that failed, died, or defaulted in a multivariate logistic regression model. Variables available were living area, economic condition, sex, age, drug resistance pattern, bacillary load, and number of treatment episodes.

\section{RESULTS}

\section{Study population}

A total of 152 EPTB patients (65 male and 87 female) were recorded within the population of randomly selected villages under Palash union council under Narsingdi district of Bangladesh. The survey regions were developing rural and sub-urban areas.

\section{Socio-economical condition of PTB patients}

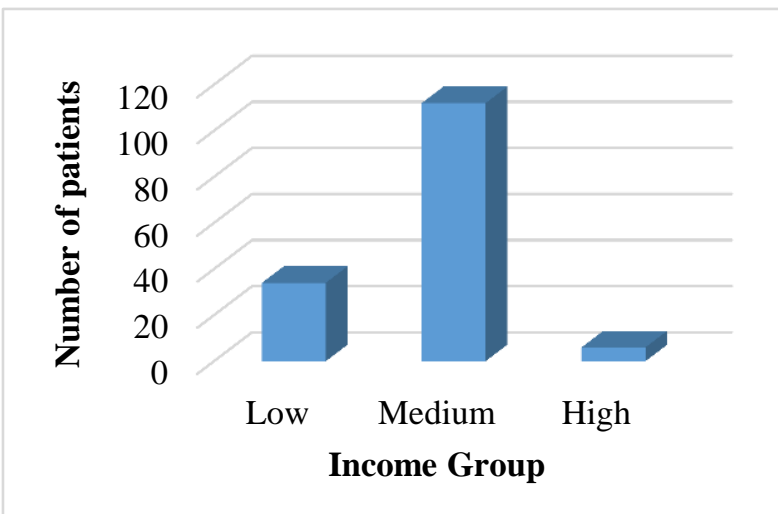

Figure 1: Socio-economic condition of PTB patients. Distribution of EPTB patients in income group $(n=152)$.

Among the EPTB patients, 34 patients were from low income group mostly of farmer (25 patients) along with a few patients from other occupation like day labor, bus driver and tailor. Medium income group population was also reported to have higher EPTB cases. A total of 112 patients were recorded in this economic group and most of them were of female sex and were housewives $(41.4 \%)$. The rest of the patients of this income group were either service holder $(15.1 \%)$ or students $(16.4 \%)$. TB cases from high income group were least reported and all those reported were business people (06 patients) (Figure $1 \& 2$ ).

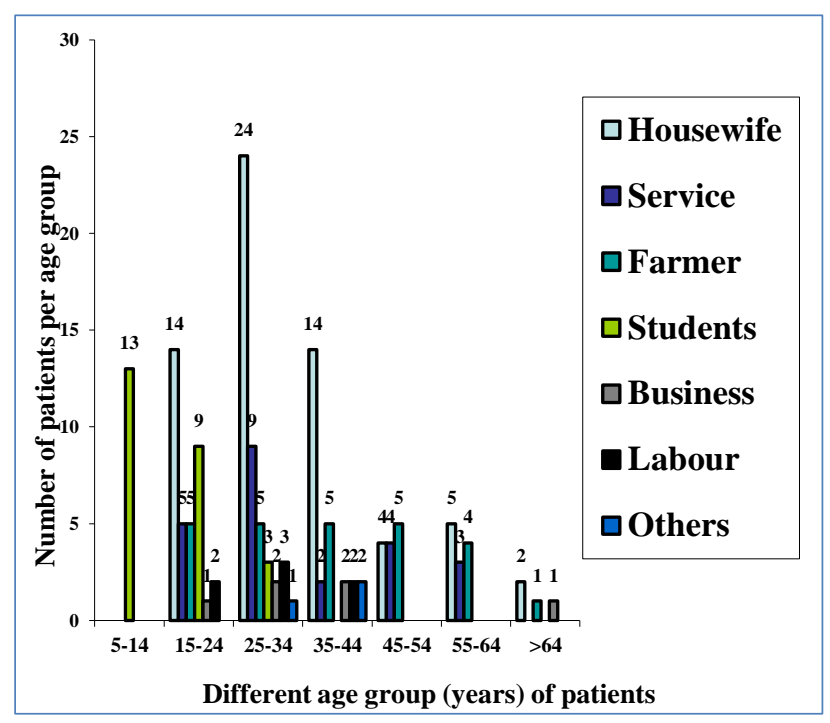

Figure 2: Distribution of patients in terms of occupation.

\section{Age \& sex differentiation of PTB patients}

The vulnerability to tuberculosis was found to vary between age and sex groups (Figure 3 ).

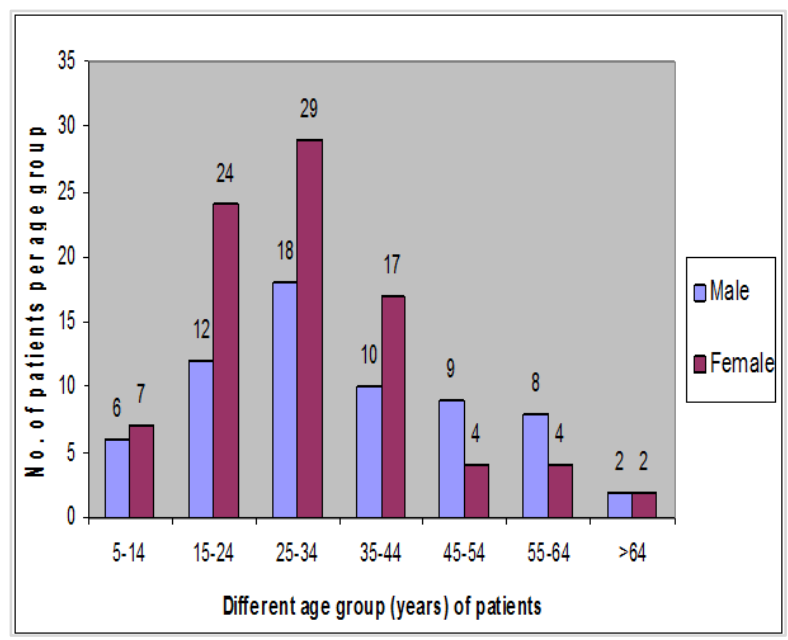

Figure 3: Sex distribution of patients of different age group.

Female were found more prone to the disease than male and the incidence of infection increased in female in older age (Figure 3 ). In the children population (aged below 14), patient's sex were not a variable in their disease vulnerability. Females were reported to have higher EPTB incidence than male of aged above 14. In the 
survey, there were a few cases of EPTB reported in children. The most vulnerable age groups were the youth working age group between aged 25 and 34 years along with the oldster population (Figure 3 ).

\section{Site of EPTB infection}

Extra pulmonary TB sites of infection was found pleural TB $29.6 \%$ patients, glands TB $24.3 \%$ patients, abdominal TB $21.0 \%$ patients, spinal TB $19.7 \%$ patients, tubercular meningitis $4.6 \%$ patients \& tubercular pericarditis $0.65 \%$ patient (Figure 4).

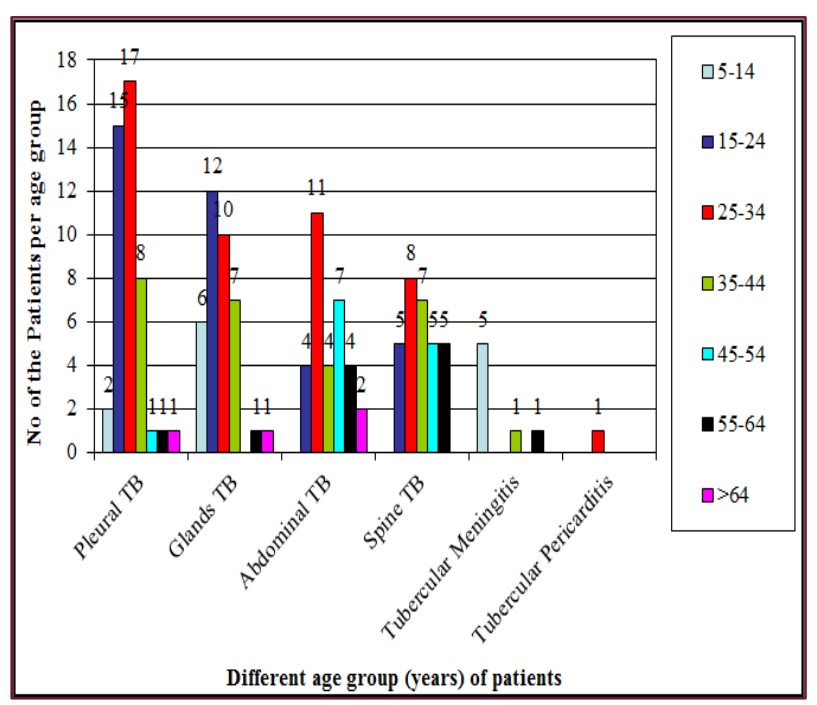

Figure 4: Site of EPTB infection.

\section{Drug resistance}

Table 1 shows drug resistance pattern of 69 strains of $M$. tuberculosis to 4 anti-tuberculosis drugs. All strains were resistant to one or more drugs. Highest resistance was found against Isoniazid (INH) either alone or in combination with other drugs. From resistance pattern of 69 strains of $M$ tuberculosis it appears that $29(42.02 \%)$ were resistant to a single drug, 21 (30.43\%) were resistant to 2 drugs, $14(20.28 \%)$ resistant to 3 drugs, 5 $(7.24 \%)$ were resistant to all 4 drugs. MDR was found in $17(24.63 \%)$ cases.
Table 1: Resistance pattern of 69 drug resistant strains of M. tuberculosis to 4 anti-tuberculosis drugs.

\begin{tabular}{|c|c|c|c|}
\hline $\begin{array}{l}\text { Number } \\
\text { of drugs }\end{array}$ & Name of drug(s) & $\begin{array}{l}\text { No. of } \\
\text { resistant } \\
\text { strains }\end{array}$ & Total \\
\hline \multirow{4}{*}{1 drug } & Isoniazid (INH) & 11 & \multirow{4}{*}{29} \\
\hline & Rifampicin (RMP) & 7 & \\
\hline & Streptomycin (SM) & 6 & \\
\hline & Ethambutol (EMB) & 5 & \\
\hline \multirow{6}{*}{2 drugs } & INH+RMP* & 5 & \multirow{6}{*}{21} \\
\hline & $\mathrm{INH}+\mathrm{SM}$ & 2 & \\
\hline & INH+EMB & 3 & \\
\hline & $\mathrm{EMB}+\mathrm{SM}$ & 4 & \\
\hline & $\mathrm{EMB}+\mathrm{RMP}$ & 3 & \\
\hline & SM+RMP & 4 & \\
\hline \multirow{3}{*}{3 drugs } & INH+RMP+SM* & 3 & \multirow{3}{*}{14} \\
\hline & INH+RMP+EMB* & 4 & \\
\hline & RMP+SM+EMB & 2 & \\
\hline 4 drugs & $\mathrm{INH}+\mathrm{RMP}+\mathrm{EMB}+\mathrm{SM} *$ & 5 & 5 \\
\hline MDR* & & & 17 \\
\hline
\end{tabular}

\section{Treatment follow-up}

In the survey, patients diagnosed to be EPTB positive were treated and follow up. All the patients were first administered with 2 or 3 months drug regime, for new cases the drug regime consisted of isoniazid, rifampicin, pyrazinamide, and ethambutol and for the patients who received at least one month TB treatment previously the drug regime consisted of isoniazid, rifampicin, pyrazinamide, streptomycin and ethambutol. Upon the regime, $86 \%$ patients were responded to the treatment and diagnosed to be cured. The treatment were completed with another drug regime; 4 or 5 months drug consisted of isoniazid and rifampicin for newly diagnosed patients and 6 or 8 months drug consisted of isoniazid, rifampicin, and ethambutol for previously treated patients. Drug intake and responses to drugs were observed at the health facility throughout the treatment. Within 6 or 8 months from starting treatment, all the 152 patients were reported to be cured. No cases of treatment failure, treatment default or death was reported among the EPTB patients in the study period.

Table 2: The follow-up of TB-patients cured after 6-months drug regimen.

\begin{tabular}{|llllllllll|}
\hline Age group (years) of patients & $\mathbf{5 - 1 4}$ & $\mathbf{1 5 - 2 4}$ & $\mathbf{2 5 - 3 4}$ & $\mathbf{3 5 - 4 4}$ & $\mathbf{4 5 - 5 4}$ & $\mathbf{5 5 - 6 4}$ & Above 64 & Total \\
\hline \multirow{2}{*}{$\begin{array}{l}\text { Patients cured after } 2 \\
\text { months of drug regimen }\end{array}$} & Male & 0 & 8 & 19 & 10 & 5 & 5 & 0 & 47 \\
\cline { 2 - 11 } & Female & 2 & 13 & 12 & 5 & 4 & 3 & 0 & 39 \\
\cline { 2 - 10 }$y$ & Sum up & 2 & 21 & 31 & 15 & 9 & 8 & 0 & 86 \\
\hline \multirow{2}{*}{$\begin{array}{l}\text { Patients cured after 5 } \\
\text { months of drug regimen }\end{array}$} & Male & 3 & 1 & 2 & 3 & 0 & 1 & 1 & 11 \\
\cline { 2 - 10 } & Female & 1 & 2 & 3 & 4 & 0 & 3 & 1 & 14 \\
\cline { 2 - 10 } & Sum up & 4 & 3 & 5 & 7 & 0 & 4 & 2 & 25 \\
\hline \multirow{2}{*}{$\begin{array}{l}\text { Patients cured after 6 } \\
\text { months of drug regimen }\end{array}$} & Male & 3 & 4 & 0 & 0 & 0 & 0 & 0 & 7 \\
\cline { 2 - 10 } & Female & 4 & 8 & 11 & 5 & 4 & 0 & 2 & 34 \\
\cline { 2 - 10 } Total & Sum up & 7 & 12 & 11 & 5 & 4 & 0 & 2 & 41 \\
\hline
\end{tabular}




\section{DISCUSSION}

Both the new and retreatment patients were included in this study. The study was based on the information collected from 152 extra-pulmonary tuberculosis (EPTB) patients, which were identified in a number of randomly selected villages among the survey area. The patients were diagnosed, received treatment with FLD regimens and treatment efficiency were investigated by follow up procedure. Living standard and economic conditions was found to influence the prevalence. EPTB had also found to infect variably with gender and in different age groups. Farmers were mostly suffered from EPTB among the low income group and female housewives among the medium income group. Females were more vulnerable than male to EPTB and it increases with ageing. The working youth and elderly people were found the most vulnerable. In drug resistance assay, $42.02 \%$ TB isolates were resistant to a single drug, $30.43 \%$ were resistant to 2 drugs, $20.28 \%$ were resistant to 3 drugs \& $7.24 \%$ were resistant to all 4 drugs (INH, RMP, SM, EMB). MDR was found in $24.63 \%$ cases.

Karim et al. ${ }^{18}$ conducted a study with 80 EPTB patient and reported that lymph node tuberculosis was $36.2 \%$ and abdominal tuberculosis was $35 \%$ and $56.3 \%$ of the patients were female. Hussain et al. ${ }^{19}$ reported that about $16 \%$ of total tuberculosis patients were EPTB and tuberculous lymphadenitis was most common in EPTB patients and $51.6 \%$ of the EPTB patients were female. Skin tuberculosis was found to be less prevalent in Bangladesh by many previous studies. Kamal et al. reported 22\% EPTB patient among 514 study patient whereas $46.9 \%$ EPTB patients were female and lymph node tuberculosis was most prevalent in their study. Rabbi et al. ${ }^{20}$ reported 33 case of abdominal tuberculosis in Bangladesh with $54.54 \%$ female patient. Sultana et al. ${ }^{21}$ reported 60 cases of cutaneous tuberculosis where $38.3 \%$ was skin tuberculosis. Talukder and Zakaria ${ }^{22}$ reported prevalence of EPTB to be $4.8 \%$. Chowdhury et al. $^{23}$ reported 99 case of extra-pulmonary tuberculosis of which ascetic fluid and pleural fluid was most prevalent site of infection.

Some previous studies conducted in Bangladesh has reported recurrence of $\mathrm{TB}$ and treatment failure in the long run with combination of drugs and increasing resistance of $M$. tuberculosis to first line drugs such as Isoniazid, rifampicin etc. ${ }^{24-27}$ In a previous study in Bangladesh by Banu et al., ${ }^{28} 88 \%$ patients had recurrent TB infection and $73.5 \% \mathrm{~TB}$ isolates were found to be multi-drug resistance. They also showed that multi-drug resistance was significantly higher among patients with previous history of anti-tuberculosis treatment. Another study in Bangladesh by Rahman et al. ${ }^{29}$ showed resistance of TB isolates for INH, RMP, EMB and SM were $76.03 \%, 71.63 \%, 27.55 \%$ and $55.65 \%$ respectively. In another study by Zaman et al., ${ }^{30}$ it was found that
48.4\% TB isolates in a rural and an urban area of Bangladesh were resistant to 1 or more drugs. Resistance to streptomycin, isoniazid, ethambutol and rifampicin was observed in $45.2 \%, 14.2 \%, 7.9 \%$ and $6.4 \%$ of isolates, respectively. Multidrug resistance was observed in $5.5 \%$ of isolates. Their study also confirms that drug resistance was significantly higher among persons who previously had received tuberculosis treatment.

In a study by Hussain et al. ${ }^{31}$ in Rajshahi, Bangladesh showed that the percentage of MDR TB in total study population was $0.4 \%$, the percentage of MDR TB among retreatment cases were $3.7 \%$ and some patients remained smear positive even after eight months of treatment. In a study conducted in Bogra district, Bangladesh registered a large number of PTB patients and $13 \%$ of them remain smear positive even after 2 years of treatment indicating rapid emergence of drug resistance in $\mathrm{TB}$ isolates ${ }^{29}$. In another study in Sunamganj district, Bangladesh showed that total resistance among new cases to any drug was $31 \%$ and for SM it was $18 \%$, INH $23 \%$, RMP $2 \%$, EMB $10 \%$ and $2 \%$ of the isolates were multidrug-resistant (MDR). ${ }^{32}$

This study in concordance with previous studies and alarms us about high prevalence of pulmonary tuberculosis in Bangladesh and increasing drug resistance in M. tuberculosis isolates. MDR TB and XDR TB percentage as well as treatment failure and recurrent $T B$ infection is increasing day by day. Based on our study results, we strongly suggest that Bangladesh rapidly scale-up access to MDR-TB diagnosis and treatment around the country, with priority for provinces with high anti-TB drug resistance prevalence.

\section{CONCLUSION}

In conclusion, MDR-EPTB patients in Bangladesh had high recurrence and death rates four years after treatment with standardized FLD regimens. Documentation of cure based on conventional smear microscopy at the end of treatment was a poor predictor of long-term outcome. Adequate identification and treatment of MDR-EPTB patients is urgently needed in order to improve long-term outcomes and to control the spread of MDR-EPTB.

\section{ACKNOWLEDGEMENTS}

We are pleased to acknowledge the Thana health complex, Palash, Narshingdi, Bangladesh for the assistance in the survey and providing information under 'Anti-tuberculosis drug resistance survey'.

Funding: No funding sources Conflict of interest: None declared

Ethical approval: The study was approved by the Upazilla health complex, Palash, Narsingdi ethics committee for TB operational research 


\section{REFERENCES}

1. Afroz H, Ali MA, Fakruddin M, Kamrunnahar, Datta S. Prevalence of multi-drug resistant (MDR) pulmonary tuberculosis in a tertiary care hospital of Narshingdi, Bangladesh. Intl J Biomed Adv Res. 2013;4(2):98-104.

2. Rahman MM. Tuberculosis: global and regional scenarios. Anwar Khan Modern Med Coll J. 2010;1(1):19-22.

3. Gonzalez OY, Adams G, Teeter LD, Bui TT, Musser JM, Graviss EA. Extrapulmonary manifestations in a large metropolitan area with a low incidence of tuberculosis. Int $\mathbf{J}$ Tuberc Lung Dis. 2003;7:1178-85.

4. Garcia-Rodriguez JF, Alvarez-Diaz H, LorenzoGarcia MV, Marino-Callejo A, Fernandez-Rial A, Sesma-Sanchez P. Extrapulmonary tuberculosis: epidemiology and risk factors. Enferm Infecc Microbiol Clín. 2011;29(7):502-9.

5. Kamal SMM, Ahasan HAMN, Ahmed S, Ayaz KFM, Mahbub MS, Khan MAI, et al. Isolation and identification of mycobacterium from extrapulmonary specimen at NTRL, NIDCH. J Med. 2010;11(2):128-30.

6. Akhter Z, Hassan H, Hanif A. Study of Frequency of extra pulmonary tuberculosis in Gulab Devi hospital Lahore. J Fatima Jinnah Med Coll. 2013;7(1):45-51

7. Golden MP, Vikram HR. Extrapulmonary tuberculosis: an overview. Am Family Physician. 2005;72(9):1761-8.

8. Lönnroth K, Raviglione M. Global epidemiology of tuberculosis: prospects for control, Sem Resp Crit Care med. 2008;29(5):481-91.

9. Cox H, Kebede Y, Allamuratova S, Ismailov G, Davletmuratova Z, Byrnes G, et al. Tuberculosis recurrence and mortality after successful treatment: impact of drug resistance. PLoS Med. 2006;3(10):e384.

10. Espinal MA, Kim SJ, Suarez PG, Kam KM, Khomenko AG, Migliori GB, et al. Standard shortcourse chemotherapy for drug-resistant tuberculosis: treatment outcomes in 6 countries. JAMA. 2000;283:2537-45.

11. Dye C, Espinal MA, Watt CJ, Mbiaga C, Williams BG. Worldwide incidence of multidrug-resistant tuberculosis. J Infect Dis. 2002;185:1197-202.

12. Shah NS, Wright A, Bai GH, Barrera L, Boulahbal F, Martín-Casabona N, et al. Worldwide emergence of extensively drug-resistant tuberculosis. Emerg Infect Dis. 2007;13:380-7.

13. WHO. Guidelines for surveillance of drug resistance in tuberculosis. In: WHO, eds. WHO Guideline. 4th ed. Geneva, Switzerland: World Health Organization; 1997: 1-83.

14. An YS, Ding BC, Zhu JH, Xing Q, He XX. Report of WHO survey on drug- resistant tuberculosis in Beijing. The $\mathbf{J}$ Chinese Antitubercul Assoc. 2007;29:475-8.
15. WHO. Treatment of tuberculosis: guidelines for national programmes. WHO, eds. WHO Guideline. 3rd ed. Geneva, Switzerland: World Health Organization; 2003: 5-108.

16. Wang GJ, Xu JY, Wang GB, Zhen XA, Gao SY, Du CM. Impact of anti-tuberculosis drug resistance on treatment outcome of pulmonary tuberculosis patients receiving directly observed treatment strategy in Henan province, China. Chinese J Tubercul Resp Dis. 2006;29:527-30.

17. Quy HT, Cobelens FG, Lan NTN, Buu TN, Lambregts CS, Borgdorff MW. Treatment outcomes by drug resistance and HIV status among tuberculosis patients in Ho Chi Minh City, Vietnam. Int J Tuberc Lung Dis. 2006;10:45-51.

18. Karim MM, Chowdhury SA, Hussain MM, Faiz MA. A clinical study on extrapulmonary tuberculosis. J Bang Coll Phys Surg. 2006;24(1):1928.

19. Hussain MW, Haque MA, Banu SA, Ekram ARMS, Rahman AF. Extrapulmonary tuberculosis: experience in Rajshahi chest disease clinic and chest disease hospital. TAJ: J Teachers Assoc. 2004;17(1):16-9.

20. Rabbi ANMA, Jahan FAJ, Aziz MM. Abdominal tuberculosis. KYAMC J. 2010;1(1):16-20.

21. Sultana A, Bhuiyan MSI, Bashar A, Islam MT, Rahman MM. Pattern of cutaneous tuberculosis among children and adolescent. Bangladesh Med Res Counc Bull. 2012;38:94-7.

22. Talukder MAS, Zakaria MM. Prevalence of Extrapulmonary tuberculosis in hospital patients. 10th ASCON. 2002;087:117.

23. Chowdhury MR, Bari SMN, Islam KS, Rakibuzzaman AGM, Afrin S. Evaluation of real time pcr for the diagnosis of extrapulmonary tuberculosis and comparison with AFB microscopy among bangladeshi population. Intl $\mathrm{J}$ Nat Sci. 2012;2(1):26-32.

24. Deun AV, Salim MA, Das AP, Bastian I, Portaels F. Results of a standardised regimen for multidrugresistant tuberculosis in Bangladesh. Int $\mathrm{J}$ Tuberc Lung Dis. 2004;8(5):560-7.

25. Amin MN, Rahman MA, Flora MS, Azad MAK. Factors associated with multidrug-resistant tuberculosis. Ibrahim Med Coll J. 2009;3(1):29-33.

26. Wadud AB, Rahman AS, Miah RA, Saleh AA. Drug resistance pattern of mycobacterium tuberculosis isolated from patients attending a referral hospital. Bang J Med Microbiol. 2009;03(01):13-7.

27. Miah MR, Ali MS, Saleh AA, Sattar H. Primary drug resistance pattern of mycobacterium tuberculosis in Dhaka, Bangladesh. Bangladesh Med Res Counc Bull. 2000;26(2):33-40.

28. Banu S, Mahmud AM, Rahman MT, Hossain A, Uddin MKM, Ahmed T, et al. Multidrug-resistant tuberculosis in admitted patients at a tertiary referral hospital of Bangladesh. PLoS One. 2012;7(7):e40545. 
29. Rahman M, Kamal SMM, Mahammed FR, Alam $\mathrm{MB}$, Ahasan H. Anti-tuberculosis drug resistance pattern among different category of tuberculosis patients. J Med. 2009;10:45-7.

30. Zaman K, Rahim Z, Yunus M, Arifeen S, Baqui A, Sack D, et al. Drug resistance of Mycobacterium tuberculosis in selected urban and rural areas in Bangladesh. Scand J Infect Dis. 2005;37:21-6.

31. Hussain MW, Haque MA, Ekram ARMS, Hossain M, Rahman MF. Multi-drug resistant tuberculosis in
Rajshahi district. TAJ: J Teachers Assoc. 2005;18(2):113-7.

32. Rahman MA, Hussain MW, Alam F, Jahan MS. Multi-drug resistant tuberculosis: experience in Bogra. TAJ: J Teachers Assoc. 2005;18(1):30-6.

DOI: $10.5455 / 2349-3933 . i j a m 20140801$

Cite this article as: Afroz H, Ali MA, Fakruddin M, Kamrunnahar, Datta S. Prevalence and treatment follow-up of drug-resistant extra-pulmonary tuberculosis in rural communities in Narshingdi, Bangladesh. Int J Adv Med 2014;1:71-7. 